\title{
NOUVELLE
}

\section{Comprendre les neurones glutamatergiques chez les mammifères: un ménage à trois}

Étienne Herzog
Department of Molecular Neurobiology, Max-PlanckInstitut für experimentele Medizin, Hermann-ReinStrasse 3, 37075 Göttingen, Allemagne.

herzog@em.mpg.de
> Les neurones communiquent entre eux en libérant dans la fente synaptique des neurotransmetteurs préalablement accumulés dans les vésicules synaptiques de leurs terminaisons. Ces transmetteurs chimiques vont alors agir sur leurs récepteurs situés sur les membranes pré- et postsynaptiques. Selon la nature des récepteurs activés, le signal transmis peut être une excitation (dépolarisation), une inhibition (hyperpolarisation) ou une modulation (cascade de seconds messagers) du neurone cible.

En 1952, P. Fatt et B. Katz ont montré que la libération de neurotransmetteurs se fait par «paquets», ou quantums [1], chaque quantum représentant le contenu d'une vésicule synaptique. Pour réaliser la libération quantique, la terminaison met en jeu un système de recyclage, d'une part, des molécules de neurotransmetteurs [2] et, d'autre part, de la membrane vésiculaire [3]. La coordination spatiale et temporelle de cette machinerie est cruciale pour assurer un bon rapport signal/bruit, en particulier lors de stimulations répétées.

Acide aminé endogène, le glutamate est un métabolite ubiquitaire du vivant. Dans le cerveau, c'est également le principal neurotransmetteur excitateur utilisé par au moins $30 \%$ des neurones. La neurotransmission par le glutamate (ou glutamatergique) est ainsi la force motrice dans tous les circuits fonctionnels du système nerveux central (régulations autonomes, boucles sensorimotrices, fonctions cognitives). Par conséquent, nombre de maladies neurologiques et psychiatriques font intervenir la transmission glutamatergique.
L'absence de marqueurs protéiques spécifiques a cependant longtemps freiné l'étude des neurones glutamatergiques [4].

\section{Les trois transporteurs vésiculaires} du glutamate

Récemment, trois sous-types de transporteurs vésiculaires du glutamate (VGLUTI, 2 et 3 ), de séquences très conservées (plus de $70 \%$ d'identité), ont été isolés et caractérisés (Figure 1) [57]. En remplissant les vésicules synaptiques, ces protéines réalisent une fonction clé pour la sécrétion de glutamate. Elles sont également les premiers marqueurs protéiques spécifiques des cellules glutamatergiques [5-10]. Les VGLUT sont enchassés dans la membrane des vésicules synaptiques par dix domaines transmembranaires (Figure IB).

Les trois VGLUT ont des propriétés biophysiques et pharmacologiques de transport vésiculaire extrêmement proches [5-10]. De plus, des expériences de transfection de cellules ou de neurones non glutamatergiques ont permis d'établir sans ambiguïté que l'expression de VGLUTl ou de VGLUT2 suffisait pour induire une libération de glutamate $[5,7,8]$. Bien que probable, l'implication directe de VGLUT3 dans la libération de glutamate reste encore à démontrer $[7,10]$. En revanche, leur répartition dans le cerveau est très complémentaire (Figure 1C). VGLUTl est exprimé principalement dans les régions les plus corticales du cerveau, qui sont le siège des fonctions cognitives et d'apprentissage moteur, alors que VGLUT2 est localisé dans les neurones glutamatergiques sous-corticaux, qui sont principa- lement impliqués dans les circuits sensorimoteurs et de régulation autonome de l'organisme. Chez l'adulte, VGLUT1 et VGLUT2 délimitent donc deux territoires qui couvrent l'ensemble des neurones glutamatergiques historiquements décrits [4]. Au cours du développement, VGLUT2 est présent très précocement et couvre en partie le territoire de VGLUTl, alors que ce dernier ne l'occupe qu'au bout de trois semaines de vie postnatale [11]. VGLUT3 (que nous avons identifié à I'Inserm U.513) présente une expression restreinte à quelques sous-populations de neurones classiquement considérés comme cholinergiques, sérotonergiques ou même GABAergiques. VGLUT3 semble donc définir un système glutamatergique de type modulateur tout à fait inattendu [10].

Si les trois VGLUT sont localisés dans des populations distinctes de neurones, leurs propriétés de remplissage des vésicules synaptiques sont peu différentes et les raisons fonctionnelles de cette diversité restent à déterminer [7].

\section{Rôles respectifs des VGLUT:}

résultats de l'invalidation du gène codant pour VGLUT1

Le gène codant pour VGLUTl a récemment été invalidé (VGLUTI ${ }^{-/-}$) dans les laboratoires de R. Edwards (UCSF, San Fransisco, USA) et de N. Brose (MaxPlanck-Institut für Experimentele Medizin, Göttingen, Allemagne) [12, 13]. Chez les animaux VGLUTI ${ }^{-/-}$, nous avons montré que l'absence de VGLUTl au cours des trois premières semaines de vie postnatale est en partie compensée par la présence ubiquitaire plus précoce de VGLUT2, les animaux VGLUTI ${ }^{-/-}$ne mourant qu'après 21 jours de vie postnatale, date à laquelle le système VGLUTl est censé être parfaitement 
fonctionnel [11]. Cependant, en appliquant des conditions d'élevage adaptées, l'équipe de R.Edwards a pu maintenir en vie un grand nombre de souris $\mathrm{VGLUTI}^{-/-}$bien au-delà de cette limite [12]. À partir du début de la troisième semaine, ces souris présentent un retard global de croissance, un aspect émacié, sont aveugles et semblent présenter des troubles neurologiques moteurs.

La mutation entraîne un effondrement de la capacité du cerveau à accumuler le glutamate dans les vésicules synaptiques. II ne reste que $20 \%$ du transport vésiculaire de glutamate dans le cerveau antérieur des souris VGLUTI ${ }^{-/-}$. Pourtant, VGLUTl ne paraît pas absolument essentiel au maintien des fonctions vitales de l'organisme. VGLUT2 assurerait donc probablement la majeure partie de la transmission glutamatergique dans les centres nerveux de régulation autonome de l'organisme [7, 9].

Le cerveau des animaux VGLUTI-/- ne semble pas être affecté sur le plan morphologique et la mise en place des systèmes 2 et 3 (voir Figure 1C) n'est pas perturbée par l'absence de VGLUTI [12, 13].
Sur le plan ultrastructural, ni le nombre, ni la taille des synapses ne sont modifiés, mais une diminution importante du nombre de vésicules synaptiques à la terminaison et l'apparition de structures membranaires tubulovésiculaires atypiques sont observées (Figure 2) [12]. Une telle observation n'a jamais été faite dans les cas d'invalidation des gènes codant pour les transporteurs vésiculaires d'amines. Elle implique une interaction directe de VGLUTl avec la machinerie de recyclage ou de biogenèse des vésicules (Figure 2) [12].

A

VGLUT3 MPFNAFDTFKEKI IKPGKEGVKNAVGDSLGILORKLDGTNE EGDA I ELS E EGRPVOTSRARAPVCDCSCCG I PKRY I IAV 80 VGLUT2 M--.-ESVKORI IAPGKEGIKNFAGKSLGOIYRVLEKKDDNRETI ELTEDGKPLEVPEKKAPLCDCTCFGLPRRY I IAI 75 VGLUT1 M-..-

VGLUT3 MSGLGFCISFGIRCNLGVA IVEMVNNSTVYVDGKPE I QTAOFNWDPETVGL I HGSFFWGY IVTOIPGGF I SNKFAANRVF 160 VGLUT2 MSGLGFC I SFGIRCNLGVA I VDMVNNST I HRGGKV I KEKAKFNWDPETVEMI HGSFFWGY I I TD I PGGY I ASRLAANRVF 155 VGLUTI MSGLGFCI SFGIRCNLGVA I VSMVNNSTTHRGGHVVVQKAQFNWDPETVGL I HGSFFWGY I VTQ I PGGF I CQKFAANRVF 147 VGLUT3 GAAIFLTSTLNMFIPSAARVHYGCVMOVRILOGLVEGVTYPACHGMWSKWAPPLERSRLATTSFCGSYAGAVVAMPLAGV 240 VGLUT2 GAA I LLTSTLNML I PSAARVHYGCV I FVR I LOGLVEGVTYPACHG IWS KWAPPLERSRLATTSFCGSYAGAVIAMPLAGI 235 VGLUTI GFAIVATSTLNMLI PSAARVHYGCV I FVRI LQGLVEGVTYPACHG IWSKWAPPLERSRLATTAFCGSYAGAVVAMPLAGV 227

VGLUT3 LVOYIGWASVFY I YGMFG I IWYMFWL LOAY ECPAVHPT I SNE ERTY I ETS I GEGANL-ASLSKFNTPWRRFFTSLPVYA I 319 VGLUT2 LVOYTGWS SVFYVYGSFGMVWYMFWLLVSY ESPAKHPT I TDE ERRY I $\varepsilon$ ES I G ESANLLGAMEKFKTPWRKFFTSMPVYA 315 VGLUT1 LVPYSGWSSVFYVYGSFG I FWYLFWLLVSY ESPALHPS I S E ERKY I EDA I G ESAKLMNPVTKFNTPWRRFFTSMPVYA 307

VGLUT3 IVANACRSWTFYLLLI SOPAYFE EVFGFA I SKVGLLSAVPHMVMT IVVP I GGOLADYLRSRK I LTTTAVRKIMNCGGFGM 399 VGLUT2 I VANFCRSWTFYLLLI I SPAYFE EVFGFE I SKVGMLSAVPHLVMT I IVP I GGO I ADFLRSKO I LSTTTVRKIMNCGGFGM 395 VGLUTI IVANFCRSWTFYLLLI SQPAYFEEVFGFE I SKVGLVSALPHLVMT I IVPI GGQIADFLRSRHIMSTTNVRKLMNCGGFGM 387

VGLUT3 EATLLLVVGFSHTKGVA I SFLVLAVGFSGFA I SGFNVNHLDIAPRYAS I LMG I SNGVGTLSGMVCPLIVGAMTKHKTRE VGLUT2 EATLLLVVGYSHTRGVA I SFLVLAVGFSGFA I SGFNVNHLD IAPRYAS I LMG I SNGVGTLSGMVCPI I VGAMTKNKSRE $\varepsilon$ 475 VGLUTI EATLLLVVGYSHSKGVAISFLVLAVGFSGFA I SGFNVNHLDIAPRYAS I LMG I SNGVGTLSGMVCPI IVGAMTKHKTRE 467

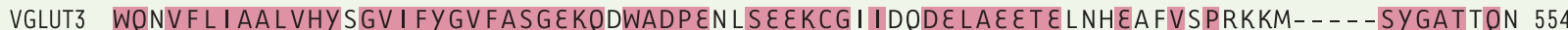

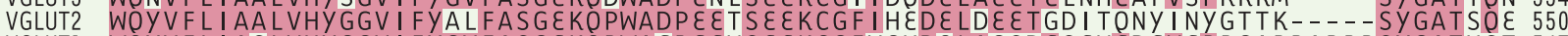
VGLUTI WQYVFLIASLVHYGGV I FYGVFASGEKQPWAEPEEMSEEKCGFVGHDQLAGSDES EMEDEVEPPGAPPAPPPSYGATHST 547

VGLUT3 CEVOKTDRROORESAFEG- EEPLSYONEEDFSETS

VGLUT2 NGGWPNGWEKKEEFVDESADDAYSYKDRDDYS

VGLUTI VQPPRPPPP - - . -

B

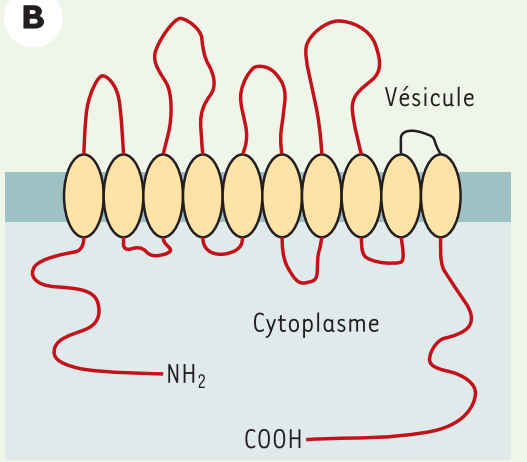

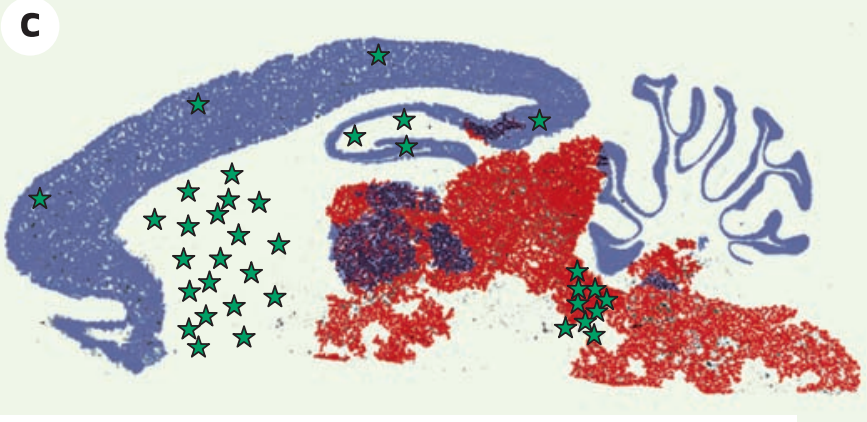

Système l (VGLUT1) @Système 2 (VGLUT2) Ł̇ Système 3 (VGLUT3)

Figure 1. Les transporteurs vésiculaires du glutamate. VGLUT1, VGLUT2 et VGLUT3 sont des protéines de 560 à 589 acides aminés présentant un très fort degré d'identité ( $A$ ), notamment dans leur partie centrale qui comprend 10 domaines transmembranaires (B). Les parties amino- et carboxyterminales, très divergentes, sont probablement cytoplasmiques. Les trois gènes présentent des profils d'expression très complémentaires dans le cerveau adulte (hybridation in situ des ARN messagers) (C). 


\section{VGLUT et contenu en glutamate} des vésicules synaptiques

Les activités électrophysiologiques des neurones dépourvus de VGLUTl ont été étudiées à partir de tranches d'hippocampe et de cervelet [12] ou de cultures unicellulaires de neurones hippocampiques [13]. Comme attendu, une partie des neurones est totalement silencieuse [13], mais une majorité présente une activité synaptique résiduelle [12, 13], vraisemblablement attribuable à l'expression ubiquitaire de VGLUT2 [11]. Cette activité résiduelle présente une forte diminution de l'amplitude du signal, de la fréquence de libération, mais aussi de la taille des quantums. La diminution de l'amplitude correspond à une diminution globale du stock de glutamate libérable, alors que la baisse de fréquence de libération s'explique par l'exocytose de vésicules vides. En effet, le cycle des vésicules synaptiques dans la membrane plasmique, mis en évidence en imagerie dynamique, est normal y compris dans les neurones totalement silencieux [3, 13]. Cette observation, combinée à la diminution du nombre de vésicules [12], suggère que les terminaisons $\mathrm{VGLUTI}^{-/-}$ont un déficit de biogenèse plutôt que de recyclage des vésicules (Figure 2). Il faut noter qu'aucun système de «contrôle qualité» ne semble empêcher les vésicules synaptiques vides de fusionner avec la membrane plasmique [13].

La diminution de la taille des quantums pourrait être expliquée par la diminution du nombre de transporteurs présents sur la vésicule (Figure 2). En effet, des expériences de surexpression de VGLUTI dans les neurones en culture ont permis de montrer que la taille des quantums peut même être supérieure à la valeur de base [13]. Ces données confirment que les transporteurs vésiculaires de glutamate travaillent en compétition avec une fuite non spécifique du glutamate vers le cytoplasme. Le point d'équilibre entre le chargement et la fuite de glutamate déterminerait la taille du quantum.

La modification de la taille du quantum libérable est l'un des nombreux mécanismes possibles de la plasticité synaptique [1]. La plasticité synaptique correspond à la modulation de la force et de l'efficacité d'une synapse en fonction de son histoire. La régulation de l'expression et/ou de l'adressage vésicu-

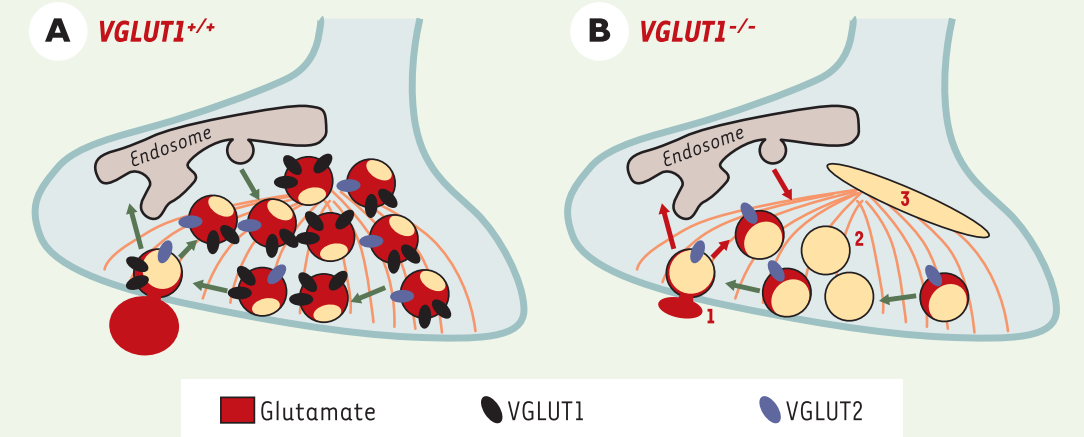

Figure 2. Modèle simplifié de terminaison nerveuse glutamatergique chez la souris. A. Pendant les trois premières semaines postnatales, la majorité des synapses glutamatergiques des neurones hippocampiques expriment VGLUTl et, de façon moindre, VGLUT2. Elles présentent un nombre élevé de vésicules synaptiques chargées en glutamate. B. L'invalidation du gène codant pour VGLUTI (VGLUT ${ }^{-/-}$) induit une diminution de la quantité de glutamate dans les vésicules (diminution du quantum libérable) (1). Certaines vésicules sont vides, mais peuvent tout de même être exocytées et le nombre de vésicules est considérablement diminué (2). Enfin, des structures tubulovésiculaires apparaissent dans la terminaison, pouvant résulter d'une altération des mécanismes de biogenèse ou de recyclage des vésicules synaptiques (3).

laire des VGLUT pourrait donc conduire à une modification de l'efficacité des synapses glutamatergiques. L'interaction originale de VGLUTI avec la machinerie de trafic membranaire est également en mesure d'altérer les propriétés de libération du glutamate. D'ailleurs, R.T. Fremeau et al. ont observé sur tranche d'hippocampe que les synapses VGLUT1 et VGLUT2 positives présentaient des formes différentes de plasticité synaptiques [12], une observation qui n'a pas été confirmée par notre groupe [13]. Ces questions sur les relations entre transport vésiculaire de glutamate et plasticité synaptique devront faire l'objet de nouvelles recherches afin d'être résolues.

\section{Conclusions}

L'invalidation du gène codant pour VGLUTI confirme que la survie d'un mammifère est possible malgré une très forte altération des systèmes cognitifs. Elle pointe également le rôle du système VGLUT2 qui semble contrôler notamment l'excitation de l'ensemble des boucles de régulations vitales pour l'organisme, dès les stades précoces du développement embryonnaire. Elle nous renseigne également sur le rôle intriguant et original de VGLUTl dans le trafic membranaire à la terminaison. Enfin, ces animaux VGLUTI ${ }^{-/-}$pourraient servir de modèle d'étude de certains phénotypes neurologiques ou psychiatriques au cours desquels une diminution de l'activité excitatrice est suspectée. À n'en pas douter, l'invalidation de VGLUT2 et VGLUT3 seront les prochaines grandes étapes dans la compréhension des systèmes de transmission glutamatergique chez les mammifères. $\diamond$

Understanding glutamatergic neurons in mammalians: « un ménage à trois »

\section{RÉFÉRENCES}

1. Burgoyne RD, Barclay JW. Splitting the quantum: Regulation of quantal release during vesicle fusion. Trends Neurosci 2002; 25: 176-8.

2. Masson J, Sagne C, Hamon M, et al. Neurotransmitter transporters in the central nervous system. Pharmacol Rev 1999; 51: 439-64. 
3. Galli T, Martinez-Arca S, Paumet F. Mécanisme de la fusion membranaire. Med Sci (Paris) 2002; 8: 1113-9.

4. Ottersen $\mathrm{OP}$, Hjelle $\mathrm{OP}$, Osen KK, et al. Amino acid transmitters in the rat nervous system. In: Paxinos $G$, ed. London: Academic Press-Elsevier, 1995: 1017-37.

5. Takamori S, Rhee JS, Rosenmund C, et al. Identification of a vesicular glutamate transporter that define a glutamatergic phenotype in neurons. Nature 2000; 407: 189-94.

6. Bellocchio દE, Reimer RJ, Fremeau RT, et al. Uptake of glutamate into synaptic vesicles by an inorganic phosphate transporter. Science 2000; 289: 957-60.

7. Fremeau RT, Voglmaier S, Seal RP, et al. VGLUTs define subsets of excitatory neurons and suggest novel roles for glutamate. Trends Neurosci 2004; 27: 98-103.
8. Takamori S, Rhee JS, Rosenmund C, et al. Identification of differentiation-associated brain-specific phosphate transporter as a second vesicular glutamate transporter (VGLUT2). J Neurosci 2001; 21: RC182.

9. Herzog $\varepsilon$, Bellenchi GC, Gras C, et al. The existence of a second vesicular glutamate transporter specifies subpopulations of glutamatergic neurons. J Neurosci 2001; 21: RC181.

10. Gras C, Herzog $\varepsilon$, Bellenchi GC, et al. A third vesicular glutamate transporter expressed by cholinergic and serotoninergic neurons. J Neurosci 2002; 22: 5442-51.
11. Miyazaki T, Fukaya M, Shimizu H, et al. Subtype switching of vesicular glutamate transporters at parallel fibre-Purkinje cell synapses in developing mouse cerebellum. Eur J Neurosci 2003; 17: 2563-72.

12. Fremeau RT, Kam K, Qureshi T, et al. Vesicular glutamate transporters 1 and 2 target to functionally distinct synaptic release sites. Science 2004; 304 : 1815-9.

13. Wojcik SM, Rhee JS, Herzog $\varepsilon$, et al. An essential role for vesicular glutamate transporter l (VGLUTl) in postnatal development and control of quantal size. Proc Natl Acad Sci USA 2004; 101: 7158-63.
NOUVELle

\section{L'amaurose congénitale de Leber: les rétinol-déshydrogénases au banc des accusés}

> Il est désormais admis que l'amaurose congénitale de Leber ( $A C L$ ) [MIM 204000] [1,2] est la dystrophie rétinienne la plus sévère et la plus précoce puisqu'elle est responsable de cécité ou de malvoyance profonde néonatale. Nous avons démontré récemment que, sous ce vocable, se distinguaient deux groupes de maladies déterminées génétiquement, se transmettant à une exception près selon le mode récessif autosomique [3]. Le premier groupe correspond à la description faite par A. Sorsby et C.E. Williams qui n'hésitaient pas à parler d'«aplasie rétinienne» [4]. Nous avons effectivement retrouvé cette entité chez des individus atteints d'une forme très sévère, très précoce et non évolutive dans laquelle l'atteinte des cônes est prédominante. Cette forme est désignée par, selon la terminologie anglosaxonne, cone-rod dystrophy. Le second groupe correspond à l'extrémité d'un spectre de gravité des rétinopathies pigmentaires et est constitué d'individus souffrant d'une dystrophie, certes sévère, mais plus progressive, débutant par une atteinte des bâtonnets (rod) et désignée selon la même terminologie par rod-cone dystrophy [5]. Pour les deux groupes, les critères cliniques d'inclusion du diagnostic sont un nystagmus congénital à grandes oscillations, une absence de poursuite oculaire, des signes digitooculaires ainsi qu'un fond d'œil normal à la naissance qui contraste avec un ERG (électrorétinogramme) plat, témoignant d'une atteinte des deux types de photorécepteurs.

La distinction entre les deux groupes cliniques se fait à la fin de la première année de vie et repose essentiellement sur: (1) l'étude du comportement de l'enfant à la
Inserm U.393, Handicaps

génétiques de l'enfant,

Hôpital Necker-Enfants

Malades, 149, rue de Sèvres,

75743 Paris Cedex 15,

France.

kaplan@necker.fr

lumière; (2) l'aspect de la rétine; (3) les données précoces de la réfraction; et (4) l'acuité visuelle (Figure l).

Jusqu'à une période très récente, 10 gènes codant pour l'ACL étaient localisés et 7 identifiés: GUCY2D [MIM 600179], RPE65 [MIM 180069], CRX [MIM 600225], AIPLI [MIM 604392], RPGRIPI [MIM 605446], CRBI [MIM 604210], TULPI [602280], LCA3 [MIM 604232] en 14q24, LCA5 [MIM 604537] en 6ql1-16 et LCA9 en 1p36 [MIM 608553]. Ces gènes sont

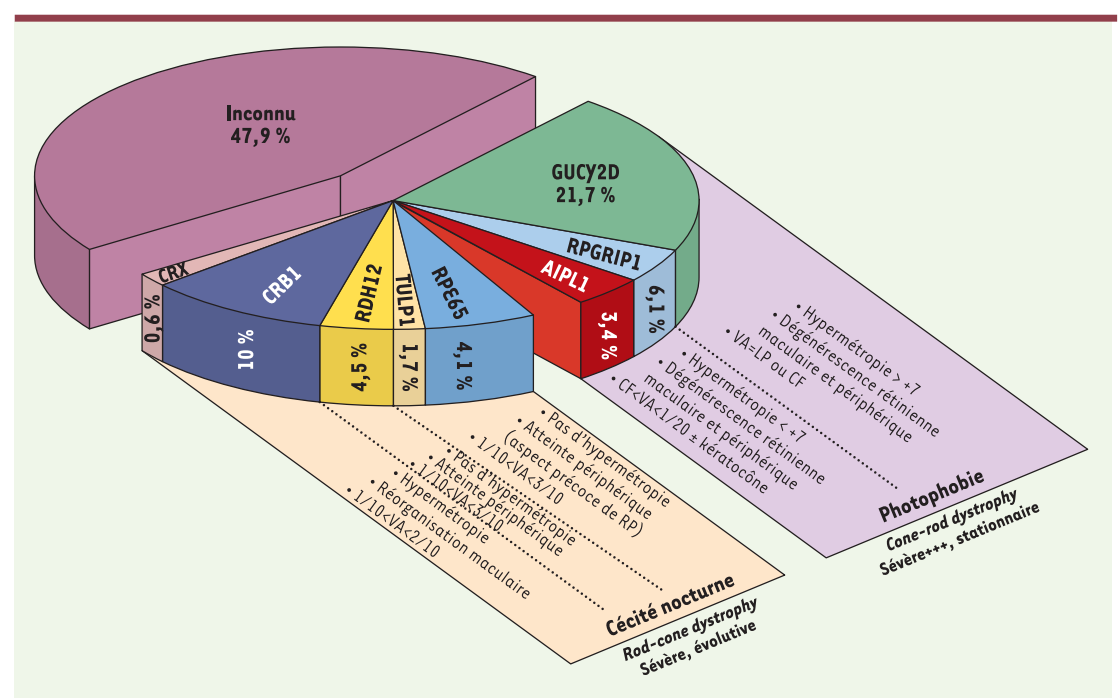

Figure 1. Représentation graphique des corrélations génotype-phénotype établies pour l'amaurose congénitale de Leber et fréquence de l'implication de chaque gène responsable de la maladie. 